doi: https://doi.org/ 10.5377/paradigma.v28i46.12839

\title{
¿Es el Tiempo de Reacción un Factor Diferenciador en el Rendimiento Académico? Un Estudio desde Kahoot
}

\section{Is Reaction Time a Differentiating Factor in Academic Performance? A Study from Kahoot}

\author{
Victor Eduardo Romero Cueva ${ }^{1}$ \\ Jimmy Rafael Landaburú Mendoza ${ }^{2}$ \\ Marlyn Elizabeth Luna Romero ${ }^{3}$ \\ Priscila Vanessa Señalín Aguirre \\ Zayda Inés Luna Romero 5
}

\begin{abstract}
Resumen
Este estudio aborda la influencia del tiempo de reacción (answer time) sobre el puntaje obtenido (score) en un test de tipo conceptual aplicado a estudiantes universitarios desde la plataforma Kahoot. El procesamiento y análisis de datos fue realizado mediante el software IBM SPSS Statistics. Para efectuar la comparación entre grupos se aplicó un ANOVA de un factor, utilizando divisiones de terciles de la variable answer time con el fin de buscar diferencias en las medias de los sujetos que finalizaron el test de forma temprana, media y tardía. Además, se realizaron correlaciones entre las variables pertinentes para contribuir a esclarecer la relación existente. Se encontró que el tiempo de reacción es importante, pero no determinante de la calidad de las respuestas. Así pues, estos hallazgos son un considerable intento para entender los fundamentos de Learning Analytics, sobre todo en tiempos de pandemia, donde la transformación digital ha mostrado ser un aliado clave, por no decir el mejor.
\end{abstract}

Palabras clave: tiempo de respuesta, puntaje, kahoot, learning analytics, test

\footnotetext{
${ }^{1}$ romervic_18@hotmail.com. Instituto Nacional de Evaluación Educativa. Quito, Ecuador. https://orcid.org/0000-0001-7314-9357

2 jlandaburu@uteq.edu.ec. Universidad Técnica Estatal de Quevedo. Quevedo, Ecuador. https://orcid.org/0000-0002-0327-343X

${ }_{3}^{3}$ marlyn.luna@ucuenca.edu.ec. Universidad de Cuenca. Cuenca, Ecuador. https://orcid.org/0000-0002-5547-6044

${ }^{4}$ psenalin@mgs.ecotec.edu.ec. Universidad Tecnológica Ecotec. https://orcid.org/0000-0001-7531-4205

${ }^{5}$ zilr.2809@gmail.com. Universidad Técnica de Machala. Machala, Ecuador. https://orcid.org/0000-0002-1025-5475
}

Recibido 1 de octubre de 2021 / Aceptado 22 de noviembre de 2021 


\begin{abstract}
This study addresses the influence of reaction time (answer time) on the performance obtained (score) in a conceptual test applied to university students from the Kahoot platform. Data processing and analysis was performed using the IBM SPSS Statistics software. To make the comparison between groups, a one-way ANOVA was carried out, using tertile divisions of the answer time variable in order to look for differences in the means of the subjects who completed the test early, mid, and late. In addition, correlations were made between the relevant variables to help clarify the existing relationship. Answer time was found to be important, but not determinant of the quality of responses. Thus, these findings are a considerable attempt to understand the fundamentals of Learning Analytics, especially in times of pandemic, where digital transformation has proven to be a key ally, if not the best.
\end{abstract}

Keywords: response time, score, kahoot, learning analytics, test

\title{
Introducción
}

Los desafíos de la instrucción remota son numerosos y van más allá de lo que se ha visto hasta ahora a causa del Covid-19, pues para Rodríguez y Luzardo (2020), uno de los mayores retos ha sido la evaluación de aprendizajes en el contexto de la virtualidad. Prácticamente todos los docentes universitarios, independientemente de la cátedra asignada, han aplicado exámenes a distancia a través de las diferentes plataformas virtuales, ante lo cual cabe plantearse la cuestión de si otorgaron el tiempo adecuado para que los examinados contesten los reactivos.

Para el BID (2020), la evaluación virtual presenta diferencias con la presencial, por lo que se genera la necesidad de desarrollar otras metodologías que respondan al contexto. Considerando que en Latinoamérica la formación y experiencia en la docencia virtual son relativamente escasas, se ha producido una deficiencia en las normativas y legalidad que cobijan los métodos de evaluación.

Como era de esperarse, la nueva modalidad ha generado preocupación en los diferentes campus universitarios. Según Nguyen, Keuseman, y Humston (2020), el cierre de los establecimientos favorece los comportamientos asociados hacia la deshonestidad académica, pues el $70 \%$ de los estudiantes universitarios ha admitido haber hecho trampa en los exámenes online. De igual forma, Balderas y Caballero-Hernández (2020) consideran que el cambio de la educación presencial a lo virtual plantea amenazas adicionales como el fraude académico durante los procesos de evaluación. Por si fuera poco, una buena parte de los estudiantes exige que las pruebas sean asincrónicas y se les asigne más tiempo para completarlas. 
De ahí que surja la necesidad de reinventar nuevos modelos de evaluación educativa que conjuguen la integridad académica con el aprendizaje online, donde el formato de la prueba y el límite de tiempo merezcan la atención de las partes involucradas, en particular sobre el tiempo de reacción o respuesta, que puede suponer un factor mucho más importante de lo que se pensaba antes de la pandemia.

Paralelo a esto, se produjo una serie de interrogantes que buscan clarificar la influencia del tiempo sobre el rendimiento académico en materia de evaluaciones virtuales: ¿Qué tipo de relación existe entre el tiempo que le toma a un estudiante contestar una prueba y el puntaje obtenido en la misma? ¿Cómo aprovechar el estudio de la relación tiempo-puntaje para promover la integridad académica? ¿Cuán pertinente es el uso de la tecnología educativa en el momento de la evaluación?

Si bien este trabajo es una respuesta a las demandas de la nueva era digital en la educación, también provee herramientas prácticas que facilitan alternativas eficaces a un problema latente a nivel de Latinoamérica, particularmente en Ecuador, como lo es la deshonestidad académica, muy posiblemente acrecentada durante las evaluaciones virtuales.

Así, por ejemplo, Flores Hinostroza et al. (2021), muestran que ciertamente hay un aumento de los niveles de fraude durante la pandemia de Covid-19 y por ello consideran que este fenómeno ha penetrado en todos los niveles de educación superior, especialmente para los exámenes virtuales, por lo cual hay que diseñar estrategias que permitan menos espacio para el engaño académico.

Otros estudios, como el de Montejo Bernardo (2020), revelaron asimismo un ligero incremento de las notas obtenidas durante el periodo de confinamiento que, es cuando se ofreció el método de evaluación no presencial. Cabe señalar que su estudio no asocia los resultados a posibles comportamientos indebidos por parte de los estudiantes, sino más bien al hecho de que la modalidad virtual permite mejorar el dominio de los contenidos, siempre y cuando se asigne una mayor cantidad de trabajo autónomo. Sin embargo, deja claro que el método de evaluación no presencial ofrece pocas garantías de integridad académica por lo cual resulta importante el ajuste del tiempo.

A este respecto, el sector de la educación ha venido improvisando soluciones para intentar mantener el normal funcionamiento de su sistema, pero aún falta mucho por hacer, principalmente en materia de evaluación. En el trabajo de García-Peñalvo et al. (2020), se presenta una hoja de ruta indicativa para el proceso de evaluación online, pero se deja en claro que no cubre todas las situaciones que pueden producirse en el contexto digital. 
Por un lado, está la posibilidad de utilizar métodos de supervisión remota (E-proctoring) y, por el otro, existe más bien la oportunidad de proteger la integridad académica sin que esta sea invasiva. Es aquí donde cobra una notable importancia Kahoot, un recurso tecnológico, que de aprovecharse adecuadamente facilitaría la promoción de la integridad académica, pero para ello es necesario entender primero la influencia del tiempo sobre el rendimiento de los estudiantes.

\section{Discusión Teórica}

Ahora bien, como es sabido por todos, la tecnología está avanzando a pasos agigantados en el mundo de hoy y con ella también lo ha hecho la educación. Para los fines de esta investigación, se presenta una descripción precisa de lo que la tecnología educativa brinda en términos de evaluación, sumado a que la aplicación de Learning Analytics - en palabras de (Rojas-Castro, 2017) - se está consolidando como un esfuerzo destinado a la mejora de las decisiones educacionales. Por ello, también es menester analizar a fondo las diferentes perspectivas sobre el impacto que genera el uso del tiempo sobre los resultados académicos que, por cierto, es la temática central de este trabajo.

Así pues, todo este bagaje tecnológico ha propiciado la incorporación de los CRS (Classroom Response Systems) en la enseñanza remota, especialmente en el ámbito de la evaluación, que, por ejemplo, representan un gran soporte para desarrollar cuestionarios y encuestas. Otros autores como Perea Moreno (2018) también les llama Sistemas de Respuesta Interactiva y asegura que además de favorecer el aprendizaje, dichos recursos aumentan la competitividad en el aula.

Algunas plataformas de este tipo que destacan en la actualidad son: Quizlet, Quizizz, Socrative y Kahoot. Esta última es precisamente la más utilizada a nivel mundial, como se señala en el trabajo de Wang y Tahir (2020), y la elegida para la experiencia de investigación que se presenta más adelante. Además de ello, Kahoot provee reportería ${ }^{6}$ que, como explican Engelhardt y Goldhammer (2019), "information about the time test-takers spend on each task is available by default nowadays in computerbased assessments". Es decir, en la actualidad existe la posibilidad de aprovechar la data que se genera en las plataformas, en este caso del tiempo de respuesta ocupado para determinada evaluación.

La mayoría de los estudios, antiguos y contemporáneos, coinciden en que existe cierta relación entre tiempo y rendimiento académico, independientemente de si esa relación es o no lineal, tal como lo indica en su manuscrito Terranova (1972). En este sentido, es lógico pensar que aquellos estudiantes que obtienen puntajes más altos sean también quienes culminan más rápido la actividad en cuestión, por ejemplo, una evaluación sumativa.

\footnotetext{
${ }^{6}$ Reportes automáticos que ciertas plataformas ofrecen para seguimiento y evaluación de resultados
} 
Hay autores como Paul y Rosenkoetter (1980) que, si bien confirman esta asociación existente, también revelan que la magnitud de correlación entre puntaje y tiempo es bastante débil.

Desde otra perspectiva, Landrum, Carlson y Manwaring (2009) pudieron constatar que el tiempo de finalización de una prueba a veces se correlaciona negativamente con el rendimiento de la prueba, pero no de manera consistente. A esto, agregaron que la edad de los estudiantes se correlacionó positivamente con el tiempo de finalización de la prueba.

Un estudio de Weber, Bohnen y Smith (2011) acerca de la relación entre la nota que un estudiante recibe en determinado examen y el tiempo que le toma completarlo, encontró que quienes lo hicieron en el mediano tiempo tendían a rendir mejor que aquellos que culminaban de forma temprana o tardía.

Por otro lado, De Paola y Gioia (2016) demostraron que el tener que realizar una prueba bajo la presión del tiempo reduce la calificación que obtienen los estudiantes, además de que las diferencias de género resultan ser grandes en magnitud y estadísticamente significativas.

En la investigación de Verleger (2016), se concluyó que no hay razón para creer que el tiempo adicional de un examen derivará en un rendimiento considerablemente mejor. En otras palabras, no existe una relación significativa entre dar tiempo adicional a los estudiantes y aumentar las calificaciones de los exámenes.

De este modo, la literatura científica revisada permite adentrarse en las distintas particularidades surgidas en el estudio del problema en mención, lo cual no solo es importante para el diseño metodológico del presente trabajo, sino que también reseña brevemente la evolución de una temática poco estudiada a lo largo del tiempo.

\section{Metodología de Investigación}

\section{Diseño}

Si bien esta investigación se clasificó de carácter exploratorio-diagnóstico, la posibilidad de utilizar reportería permitió que su alcance fuese también correlacional y explicativo.

\section{Materiales y Métodos}

Para este estudio, se hizo un muestreo intencional (no probabilístico) en la Universidad Técnica Estatal de Quevedo (UTEQ), donde participaron estudiantes que tenían características similares - esto es, todos ellos cursaban el mismo semestre académico (primer ciclo) y habían sido 
previamente entrenados en el manejo y funcionamiento de Kahoot. Además, cabe resaltar que esta fue una actividad culminante en el semestre posterior al que coincidió con el inicio de la pandemia.

De esta forma, fue posible estudiar la influencia del tiempo de respuesta (answer time) sobre el puntaje obtenido (score) en un test de tipo conceptual (Desarrollo de Habilidades del Pensamiento) que comprendió 15 preguntas, cada una con una duración máxima de 20 segundos. Este test (examen final) fue aplicado desde la plataforma Kahoot, que de hecho fue la herramienta online utilizada para la recolección de datos.

\section{Procesamiento y Análisis de la Información}

Una vez llevada a cabo la evaluación, se procede a descargar el reporte, el cual es un libro de Excel que viene en formato.xlsx, en donde se muestra una hoja de cálculo denominada RawReportData. Esta hoja presenta las variables indicadas en la Figura 1, de las cuales Total Score (points), Score without Answer Streak Bonus (points) y Answer Time (seconds) son de interés para este estudio.

Luego, para el procesamiento de los datos extraídos de Kahoot se utilizó principalmente el software IBM SPSS Statistics. En un primer análisis se obtuvieron los estadísticos descriptivos. Para efectuar la comparación entre grupos se realizó un ANOVA de un factor, utilizando divisiones de terciles de la variable answer time con el fin de buscar diferencias en las medias de los sujetos que finalizaron el test de forma temprana, media y tardía.

\section{Figura 1}

Data descargada

\begin{tabular}{|c|c|c|c|c|c|c|c|c|c|}
\hline 1 & Correct & Incorrect & & Score (points) & & $\begin{array}{l}\text { Score without Answer Streak } \\
\text { Bonus (points) }\end{array}$ & Current Total Score (points) & Answer Time (\%) & Answer Time (seconds) \\
\hline 2 & 1 & & 0 & & 550 & 550 & 550 & $90.00 \%$ & 18,0 \\
\hline 3 & 0 & & 1 & & 0 & 0 & 0 & $95.00 \%$ & 19,0 \\
\hline 4 & 0 & & 1 & & 0 & 0 & 0 & $89.00 \%$ & 17,8 \\
\hline 5 & 0 & & 1 & & 0 & 0 & 0 & $57.00 \%$ & 11,4 \\
\hline 6 & 1 & & 0 & & 598 & 598 & 598 & $80.50 \%$ & 16,1 \\
\hline 7 & 0 & & 1 & & 0 & 0 & 0 & $8200 \%$ & 16,4 \\
\hline 8 & 0 & & 1 & & 0 & 0 & 0 & $84.00 \%$ & 16,8 \\
\hline 9 & 0 & & 1 & & 0 & 0 & 0 & $80.00 \%$ & 16,0 \\
\hline 10 & 1 & & 0 & & 610 & 610 & 610 & $78.00 \%$ & 15,6 \\
\hline 11 & 1 & & 0 & & 830 & 830 & 890 & $34.00 \%$ & 6.8 \\
\hline 12 & 1 & & 0 & & 948 & 848 & 1498 & $30.50 \%$ & 6,1 \\
\hline 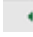 & \begin{tabular}{ll|l}
1 & 11 \\
\end{tabular} & 12 Quiz & & 14 Quiz & & RawReportData Data & (4) : 4 & & \\
\hline
\end{tabular}

Nota. Fuente: Kahoot/Excel - Captura de pantalla. 
Por otra parte, también se realizaron correlaciones entre las variables mencionadas para contribuir a esclarecer la relación existente.

\section{Resultados}

\section{Influencia del Tiempo sobre el Puntaje}

\section{Resultados Descriptivos}

En esta sección del trabajo se hace hincapié en que puede existir cierta asociación entre tiempo y puntaje. Por esta razón, Total Score (points) -el puntaje obtenido- se presenta como la variable dependiente (ver Tabla 1).

\section{Tabla 1}

Descriptivos de la variable dependiente

\begin{tabular}{|c|c|c|c|c|}
\hline Variable & Estadístico & & & Desv. Error \\
\hline \multirow[t]{13}{*}{ Total Score (points) } & Media & & 5195,59 & 170,034 \\
\hline & $\begin{array}{l}95 \% \text { de intervalo de } \\
\text { confianza para la media }\end{array}$ & Límite inferior & 4858,72 & \\
\hline & & Límite superior & 5532,46 & \\
\hline & Media recortada al 5\% & & 5167,34 & \\
\hline & Mediana & & 4934,00 & \\
\hline & Varianza & & 3295926,174 & \\
\hline & Desv. Desviación & & 1815,469 & \\
\hline & Mínimo & & 1640 & \\
\hline & Máximo & & 9341 & \\
\hline & Rango & & 7701 & \\
\hline & Rango intercuartil & & 2525 & \\
\hline & Asimetría & &, 265 &, 226 \\
\hline & Curtosis & &,- 688 & ,449 \\
\hline
\end{tabular}

Nota. Fuente: SPSS - Elaboración propia.

En la Figura 2 se observa una buena aproximación a la normalidad de la variable dependiente Total Score (points). Es decir, los cuantiles muestrales (Valor observado) parecen ajustarse razonablemente a los cuantiles teóricos (Normal esperado). No obstante, con el fin de aumentar la rigurosidad, se recurre a procedimientos analíticos. 


\section{Figura 2}

Gráfico Q-Q normal de Total Score (points)

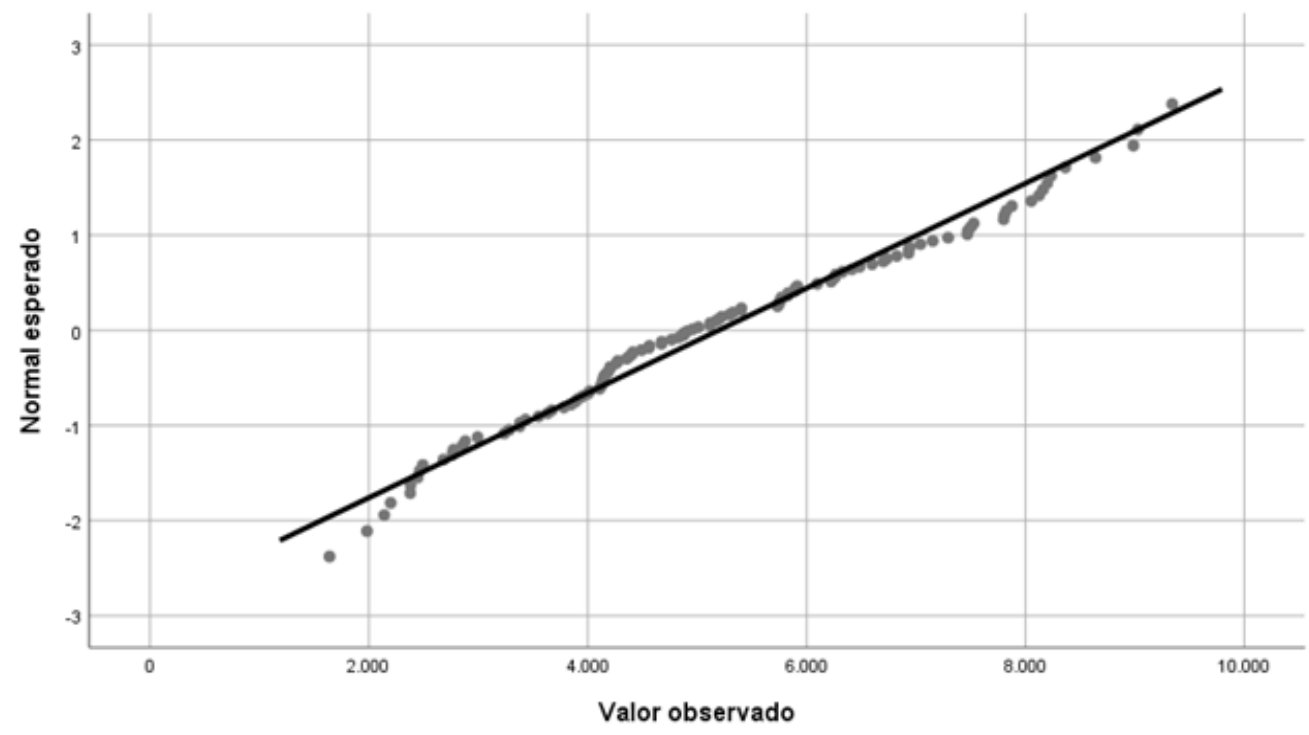

Nota. Fuente: SPSS - Elaboración propia.

Como se advirtió antes, se utilizó una prueba estadística para el respectivo contraste de hipótesis. Aquí, como se sabe, se dispone de la prueba Kolmogorov-Smirnov (No Paramétrica) para la normalidad. En la Tabla 2, se puede observar que la probabilidad asociada desde la perspectiva de la hipótesis nula (de normalidad) es 0,076. Por tanto, se acepta dicha hipótesis.

\section{Tabla 2}

Pruebas de normalidad

\begin{tabular}{cccccccc}
\hline Variable & \multicolumn{6}{c}{ Pruebas de hipótesis } \\
\hline & \multicolumn{2}{c}{ Kolmogorov-Smirnov ${ }^{\text {a }}$} & \multicolumn{3}{c}{ Shapiro-Wilk } \\
\cline { 2 - 7 } & Estadístico & gl & Sig. & Estadístico & gl & Sig. \\
Total Score (points) &, 079 & 114 &, 076 &, 977 & 114 &, 050 \\
\hline
\end{tabular}

a. Corrección de significación de Lilliefors

Nota. Fuente: SPSS - Elaboración propia.

En la Figura 3 puede observarse que las barras de error se solapan, lo cual podría hacer pensar que no existe diferencia significativa entre los valores medios de los grupos estudiados. Sin embargo, hay que evaluarlo a través de procedimientos analíticos, como la prueba ANOVA. 


\section{Figura 3}

Barras de error

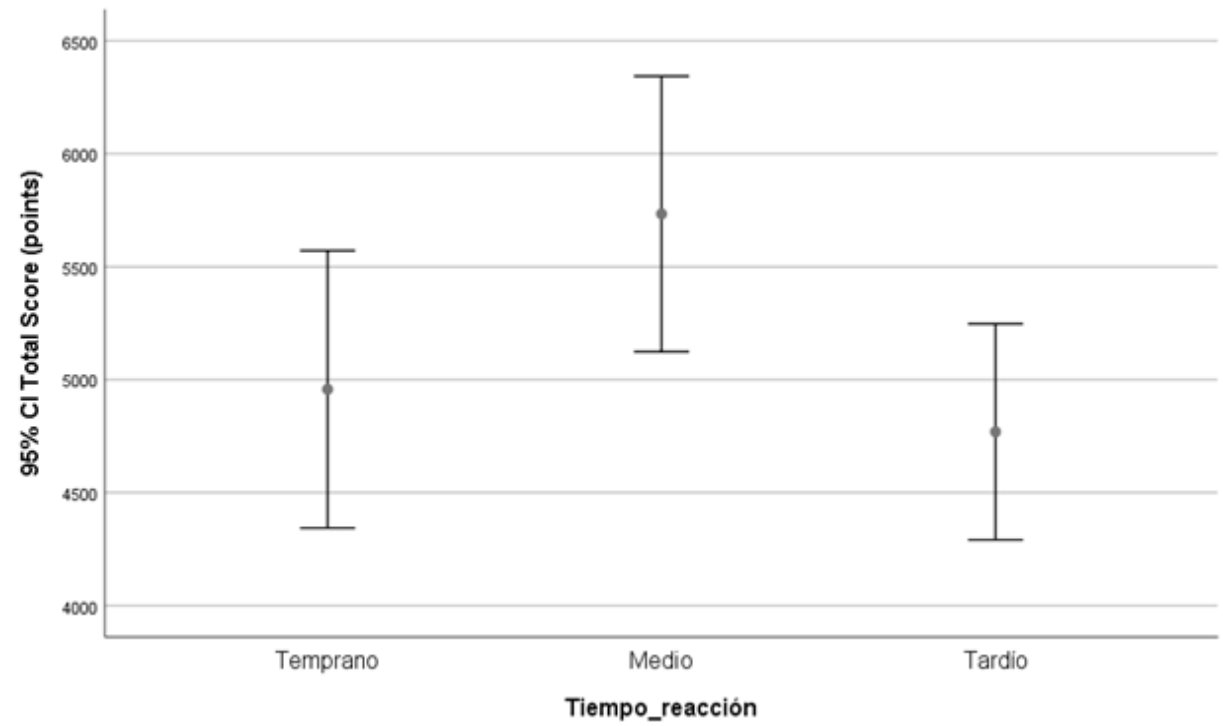

Nota. Fuente: SPSS - Elaboración propia.

Como se mencionó en la metodología, la variable answer time, al ser de naturaleza continua (medida en segundos), tuvo que ser categorizada a través de divisiones de terciles (ver Tabla 3). Por ello, es que se presenta el tiempo en forma de tres nuevas categorías: temprano, medio, tardío. Esto, con el fin de facilitar el análisis ANOVA.

\section{Tabla 3}

Descriptivos de la variable categorizada

\begin{tabular}{|c|c|c|c|c|c|c|c|c|}
\hline \multirow[b]{2}{*}{$\begin{array}{c}\text { Categorías } \\
\text { Total Score } \\
\text { (points) }\end{array}$} & \multirow[b]{2}{*}{$\mathrm{N}$} & \multirow[b]{2}{*}{ Media } & \multirow[b]{2}{*}{$\begin{array}{l}\text { Desv. } \\
\text { Desviación }\end{array}$} & \multicolumn{3}{|c|}{$\begin{array}{c}95 \% \text { de intervalo de } \\
\text { confianza para la media }\end{array}$} & \multirow[b]{2}{*}{ Mínimo } & \multirow[b]{2}{*}{ Máximo } \\
\hline & & & & $\begin{array}{l}\text { Desv. } \\
\text { error }\end{array}$ & $\begin{array}{l}\text { Límite } \\
\text { inferior }\end{array}$ & $\begin{array}{l}\text { Límite } \\
\text { superior }\end{array}$ & & \\
\hline Temprano & 38 & 4957,18 & 1867,217 & 302,903 & 4343,45 & 5570,92 & 1640 & 8364 \\
\hline Medio & 43 & 5733,56 & 1980,199 & 301,977 & 5124,14 & 6342,97 & 1983 & 9341 \\
\hline Tardío & 33 & 4769,12 & 1347,950 & 234,648 & 4291,16 & 5247,08 & 2493 & 7825 \\
\hline Total & 114 & 5195,59 & 1815,469 & 170,034 & 4858,72 & 5532,46 & 1640 & 9341 \\
\hline
\end{tabular}

Nota. Fuente: SPSS - Elaboración propia. 
La Tabla 4 provee información importante para determinar si existen diferencias estadísticamente significativas entre las medias de los tres grupos.

H0: las medias de los grupos son iguales. $\mu 1=\mu 2=\ldots=\mu \mathrm{k}$

$\mathrm{H} 1$ : alguna de las medias es distinta. $\mu \mathrm{i} \neq \mu \mathrm{j}$ para algún i y j

En este caso, dado el nivel de significancia mostrado, se rechaza la hipótesis nula y se confirma que hay al menos dos medias grupales que son diferentes entre sí. Es decir, al menos una de las variables independientes influye en la respuesta Y (Score).

\section{Tabla 4}

Prueba ANOVA

Total Score (points)

\begin{tabular}{lccccc}
\hline & Suma de cuadrados & Gl & Media cuadrática & F & Sig. \\
\hline Entre grupos & 20606331,792 & 2 & 10303165,896 & 3,251 &, 042 \\
Dentro de grupos & 351833325,830 & 111 & 3169669,602 & & \\
\hline Total & 372439657,623 & 113 & & & \\
\hline
\end{tabular}

Nota. Fuente: SPSS - Elaboración propia.

Ahora bien, para conocer qué grupos específicos son diferentes, se realizan pruebas de comparación múltiple post hoc indicadas en la Tabla 5.

\section{Tabla 5}

Pruebas Post-hoc

Variable dependiente: Total Score (points)

Intervalo de confianza al $95 \%$

\begin{tabular}{cccccccc} 
Pruebas & $\begin{array}{c}\text { (I)Tiempo } \\
\text { de reacción }\end{array}$ & $\begin{array}{c}\text { (J)Tiempo } \\
\text { de reacción }\end{array}$ & $\begin{array}{c}\text { Diferencia de } \\
\text { medias (I-J) }\end{array}$ & Desv. Error & Sig. & $\begin{array}{c}\text { Límite } \\
\text { inferior }\end{array}$ & $\begin{array}{c}\text { Límite } \\
\text { superior }\end{array}$ \\
\hline \multirow{2}{*}{ Temprano } & Medio & $-776,374$ & 396,391 &, 127 & $-1718,03$ & 165,28 \\
& & Tardío & 188,063 & 423,631 &, 897 & $-818,30$ & 1194,42 \\
HSD & \multirow{2}{*}{ Medio } & Temprano & 776,374 & 396,391 &, 127 & $-165,28$ & 1718,03 \\
& & Tardío & 964,437 & 412,024 &, 054 & $-14,35$ & 1943,23 \\
& \multirow{2}{*}{ Tardío } & Temprano & $-188,063$ & 423,631 &, 897 & $-1194,42$ & 818,30 \\
& & Medio & $-964,437$ & 412,024 &, 054 & $-1943,23$ & 14,35 \\
\hline
\end{tabular}




\begin{tabular}{ccccccccc}
\hline & & & & & \multicolumn{3}{c}{ Intervalo de confianza } \\
Pruebas & & & & & \multicolumn{2}{c}{ al 95\% } \\
& $\begin{array}{c}\text { (I)Tiempo } \\
\text { de reacción }\end{array}$ & $\begin{array}{c}\text { (J)Tiempo } \\
\text { de reacción }\end{array}$ & $\begin{array}{c}\text { Diferencia de } \\
\text { medias (I-J) }\end{array}$ & Desv. Error & Sig. & $\begin{array}{c}\text { Límite } \\
\text { inferior }\end{array}$ & $\begin{array}{c}\text { Límite } \\
\text { superior }\end{array}$ \\
\hline \multirow{2}{*}{ Temprano } & Medio & $-776,374$ & 427,715 &, 171 & $-1798,13$ & 245,39 \\
& & Tardío & 188,063 & 383,157 &, 876 & $-730,36$ & 1106,48 \\
Games- & \multirow{2}{*}{ Medio } & Temprano & 776,374 & 427,715 &, 171 & $-245,39$ & 1798,13 \\
& & Tardío & $964,437 *$ & 382,427 &, 036 & 49,52 & 1879,35 \\
& \multirow{2}{*}{ Tardío } & Temprano & $-188,063$ & 383,157 &, 876 & $-1106,48$ & 730,36 \\
& & Medio & $-964,437 *$ & 382,427 &, 036 & $-1879,35$ & $-49,52$ \\
\hline
\end{tabular}

*. La diferencia de medias es significativa en el nivel 0,05.

Nota. Fuente: SPSS - Elaboración propia.

Se observa que básicamente hay diferencias en el rendimiento (score) entre los sujetos que completaron el test de forma media y tardía, lo que también puede comprobarse mediante el gráfico de medias que se muestra en la Figura 4.

\section{Figura 4}

Gráfico de medias

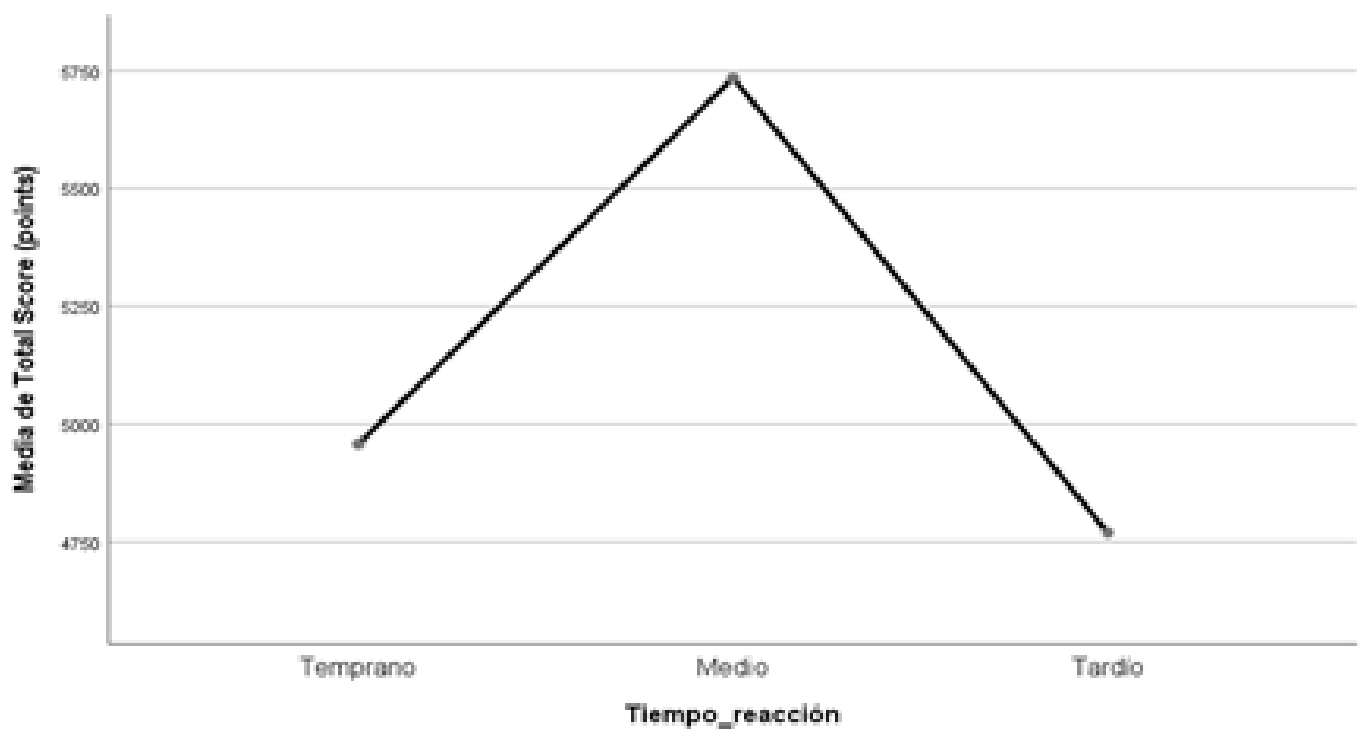

Nota. Fuente: SPSS - Elaboración propia. 


\section{Resultados Correlaciones}

Es importante conocer que para este análisis se seleccionó la variable Score without Answer Streak Bonus (points) para evitar cualquier tipo de sesgo en los resultados, puesto que Kahoot calcula los puntos obtenidos de una forma muy particular:

- Todas las preguntas valen 1000 puntos si son respondidas por debajo de 0.5 segundos.

- Para calcular los puntos se utiliza la fórmula:

Redondear $(1000 *(1-(([$ tiempo de respuesta] / [tiempo predefinido]) / 2)) ).

Es decir, al utilizar esta variable, se evita correlacionar el tiempo (en segundos) con puntajes compuestos por alguna bonificación extra.

Los descriptivos presentados en las Tablas 6 y 7, muestran diferentes rendimientos y tiempos de reacción, lo cual permite aproximar qué preguntas podrían resultar fáciles o difíciles.

Por ejemplo, la pregunta dos (2 Quiz) se podría considerar muy fácil, puesto que, en promedio, es aquí donde los sustentantes logran el puntaje más alto utilizando el menor tiempo posible. Cosa que no ocurre con la pregunta nueve (9 Quiz) donde, en promedio, los sustentantes ocupan el mayor tiempo posible y obtienen uno de los puntajes más bajos.

Sin intentar profundizar en estos comportamientos, puesto que para eso existe la psicometría, se busca mostrar al docente una forma accesible de valorar preguntas del tipo conceptual.

\section{Tabla 6}

Descriptivos por pregunta 1-8

\begin{tabular}{clccc}
\hline $\begin{array}{c}\text { Question } \\
\text { Number }\end{array}$ & \multicolumn{1}{c}{ Variable } & Media & $\begin{array}{c}\text { Desv. } \\
\text { Desviación }\end{array}$ & N \\
\hline \multirow{2}{*}{ 1 Quiz } & Score without Answer Streak Bonus (points) & 271,98 & 371,326 & 144 \\
& Answer Time (seconds) & 11,579 & 6,5245 & 144 \\
& Score without Answer Streak Bonus (points) & 756,40 & 306,914 & 144 \\
\multirow{2}{*}{ Quiz } & Answer Time (seconds) & 5,456 & 3,8576 & 144 \\
& Score without Answer Streak Bonus (points) & 358,10 & 402,393 & 144 \\
& Answer Time (seconds) & 10,449 & 5,4030 & 144 \\
& Score without Answer Streak Bonus (points) & 377,50 & 405,273 & 144 \\
& Answer Time (seconds) & 10,210 & 5,7313 & 144 \\
\hline
\end{tabular}


Continúa Tabla 6

\begin{tabular}{|c|c|c|c|c|}
\hline $\begin{array}{l}\text { Question } \\
\text { Number }\end{array}$ & Variable & Media & $\begin{array}{c}\text { Desv. } \\
\text { Desviación }\end{array}$ & $\mathbf{N}$ \\
\hline \multirow{2}{*}{5 Quiz } & Score without Answer Streak Bonus (points) & 369,15 & 384,863 & 144 \\
\hline & Answer Time (seconds) & 10,206 & 5,1252 & 144 \\
\hline \multirow{2}{*}{6 Quiz } & Score without Answer Streak Bonus (points) & 507,72 & 379,390 & 144 \\
\hline & Answer Time (seconds) & 9,699 & 4,6183 & 144 \\
\hline \multirow{2}{*}{7 Quiz } & Score without Answer Streak Bonus (points) & 247,10 & 344,944 & 144 \\
\hline & Answer Time (seconds) & 11,007 & 4,9096 & 144 \\
\hline \multirow{2}{*}{8 Quiz } & Score without Answer Streak Bonus (points) & 227,62 & 324,357 & 144 \\
\hline & Answer Time (seconds) & 13,140 & 4,9435 & 144 \\
\hline
\end{tabular}

Nota. Fuente: SPSS - Elaboración propia.

\section{Tabla 7}

Descriptivos por pregunta 9-15

\begin{tabular}{|c|c|c|c|c|}
\hline $\begin{array}{l}\text { Question } \\
\text { Number }\end{array}$ & Variable & Media & $\begin{array}{c}\text { Desv. } \\
\text { Desviación }\end{array}$ & $\mathrm{N}$ \\
\hline \multirow{2}{*}{9 Quiz } & Score without Answer Streak Bonus (points) & 218,86 & 315,604 & 144 \\
\hline & Answer Time (seconds) & 14,465 & 4,6650 & 144 \\
\hline \multirow{2}{*}{10 Quiz } & Score without Answer Streak Bonus (points) & 334,75 & 342,425 & 144 \\
\hline & Answer Time (seconds) & 13,815 & 4,2122 & 144 \\
\hline \multirow{2}{*}{11 Quiz } & Score without Answer Streak Bonus (points) & 325,34 & 349,541 & 144 \\
\hline & Answer Time (seconds) & 13,626 & 4,7440 & 144 \\
\hline \multirow{2}{*}{12 Quiz } & Score without Answer Streak Bonus (points) & 341,98 & 341,622 & 144 \\
\hline & Answer Time (seconds) & 14,287 & 4,3425 & 144 \\
\hline \multirow{2}{*}{13 Quiz } & Score without Answer Streak Bonus (points) & 152,61 & 303,187 & 144 \\
\hline & Answer Time (seconds) & 12,189 & 4,9064 & 144 \\
\hline \multirow{2}{*}{14 Quiz } & Score without Answer Streak Bonus (points) & 456,57 & 376,195 & 144 \\
\hline & Answer Time (seconds) & 11,426 & 5,5709 & 144 \\
\hline \multirow{2}{*}{15 Quiz } & Score without Answer Streak Bonus (points) & 174,57 & 320,581 & 144 \\
\hline & Answer Time (seconds) & 11,628 & 5,2440 & 144 \\
\hline
\end{tabular}

Nota. Fuente: SPSS - Elaboración propia. 
Los resultados en las Tablas 8, 9, 19 y 11, muestran una relación negativa entre las dos variables de todas las preguntas analizadas que, era algo que se esperaba. Además, casi todas las correlaciones resultan ser significativas $(<0.05)$, con excepción de las preguntas siete (7 Quiz) y ocho (8 Quiz). Respecto a los coeficientes, si bien no son tan altos, tampoco puede decirse que sean extremadamente bajos, lo cual tal vez se deba al tamaño de la muestra. En definitiva, existe cierta asociación entre las dos variables estudiadas.

\section{Tabla 8}

Correlaciones por pregunta 1-4

\begin{tabular}{|c|c|c|c|c|}
\hline $\begin{array}{l}\text { Question } \\
\text { Number }\end{array}$ & & & $\begin{array}{l}\text { Score without Answer } \\
\text { Streak Bonus (points) }\end{array}$ & $\begin{array}{l}\text { Answer Time } \\
\text { (seconds) }\end{array}$ \\
\hline \multirow{6}{*}{ 1 Quiz } & \multirow{3}{*}{$\begin{array}{l}\text { Score without Answer } \\
\text { Streak Bonus (points) }\end{array}$} & Correlación de Pearson & 1 &,$- 292 * *$ \\
\hline & & Sig.(bilateral) & & ,000 \\
\hline & & $\mathrm{N}$ & 144 & 144 \\
\hline & \multirow{3}{*}{ Answer Time (seconds) } & Correlación de Pearson &,$- 292 * *$ & 1 \\
\hline & & Sig. (bilateral) &, 000 & \\
\hline & & $\mathrm{N}$ & 144 & 144 \\
\hline \multirow{6}{*}{2 Quiz } & \multirow{3}{*}{$\begin{array}{l}\text { Score without Answer } \\
\text { Streak Bonus (points) }\end{array}$} & Correlación de Pearson & 1 &,$- 418 * *$ \\
\hline & & Sig. (bilateral) & & ,000 \\
\hline & & $\mathrm{N}$ & 144 & 144 \\
\hline & \multirow{3}{*}{ Answer Time (seconds) } & Correlación de Pearson &,$- 418^{* *}$ & 1 \\
\hline & & Sig. (bilateral) &, 000 & \\
\hline & & $\mathrm{N}$ & 144 & 144 \\
\hline \multirow{6}{*}{3 Quiz } & \multirow{3}{*}{$\begin{array}{l}\text { Score without Answer } \\
\text { Streak Bonus (points) }\end{array}$} & Correlación de Pearson & 1 &,$- 451 * *$ \\
\hline & & Sig. (bilateral) & &, 000 \\
\hline & & $\mathrm{N}$ & 144 & 144 \\
\hline & \multirow{3}{*}{ Answer Time (seconds) } & Correlación de Pearson &,$- 451 * *$ & 1 \\
\hline & & Sig. (bilateral) &, 000 & \\
\hline & & $\mathrm{N}$ & 144 & 144 \\
\hline \multirow{6}{*}{4 Quiz } & \multirow{3}{*}{$\begin{array}{l}\text { Score without Answer } \\
\text { Streak Bonus (points) }\end{array}$} & Correlación de Pearson & 1 &,$- 423^{* *}$ \\
\hline & & Sig. (bilateral) & &, 000 \\
\hline & & $\mathrm{N}$ & 144 & 144 \\
\hline & \multirow{3}{*}{ Answer Time (seconds) } & Correlación de Pearson &,$- 423 * *$ & 1 \\
\hline & & Sig. (bilateral) &, 000 & \\
\hline & & $\mathrm{N}$ & 144 & 144 \\
\hline
\end{tabular}

Nota. Fuente: SPSS - Elaboración propia. 


\section{Tabla 9}

Correlaciones por pregunta 5-8

\begin{tabular}{|c|c|c|c|c|}
\hline $\begin{array}{l}\text { Question } \\
\text { Number }\end{array}$ & & & $\begin{array}{l}\text { Score without Answer } \\
\text { Streak Bonus (points) }\end{array}$ & $\begin{array}{c}\text { Answer Time } \\
\text { (seconds) }\end{array}$ \\
\hline \multirow{6}{*}{5 Quiz } & \multirow{3}{*}{$\begin{array}{l}\text { Score without Answer } \\
\text { Streak Bonus (points) }\end{array}$} & Correlación de Pearson & 1 &,$- 169^{*}$ \\
\hline & & Sig.(bilateral) & &, 042 \\
\hline & & $\mathrm{N}$ & 144 & 144 \\
\hline & \multirow{3}{*}{ Answer Time (seconds) } & Correlación de Pearson &,$- 169 *$ & 1 \\
\hline & & Sig. (bilateral) &, 042 & \\
\hline & & $\mathrm{N}$ & 144 & 144 \\
\hline \multirow{6}{*}{6 Quiz } & \multirow{3}{*}{$\begin{array}{l}\text { Score without Answer } \\
\text { Streak Bonus (points) }\end{array}$} & Correlación de Pearson & 1 &,$- 367 * *$ \\
\hline & & Sig. (bilateral) & &, 000 \\
\hline & & $\mathrm{N}$ & 144 & 144 \\
\hline & \multirow{3}{*}{ Answer Time (seconds) } & Correlación de Pearson &,$- 367 * *$ & 1 \\
\hline & & Sig. (bilateral) &, 000 & \\
\hline & & $\mathrm{N}$ & 144 & 144 \\
\hline \multirow{6}{*}{7 Quiz } & \multirow{3}{*}{$\begin{array}{l}\text { Score without Answer } \\
\text { Streak Bonus (points) }\end{array}$} & Correlación de Pearson & 1 &,- 001 \\
\hline & & Sig. (bilateral) & & ,986 \\
\hline & & $\mathrm{N}$ & 144 & 144 \\
\hline & \multirow{3}{*}{ Answer Time (seconds) } & Correlación de Pearson &,- 001 & 1 \\
\hline & & Sig. (bilateral) & ,986 & \\
\hline & & $\mathrm{N}$ & 144 & 144 \\
\hline \multirow{6}{*}{8 Quiz } & \multirow{3}{*}{$\begin{array}{l}\text { Score without Answer } \\
\text { Streak Bonus (points) }\end{array}$} & Correlación de Pearson & 1 &,- 085 \\
\hline & & Sig. (bilateral) & & ,311 \\
\hline & & $\mathrm{N}$ & 144 & 144 \\
\hline & \multirow{3}{*}{ Answer Time (seconds) } & Correlación de Pearson &,- 085 & 1 \\
\hline & & Sig. (bilateral) &, 311 & \\
\hline & & $\mathrm{N}$ & 144 & 144 \\
\hline
\end{tabular}

Nota. Fuente: SPSS - Elaboración propia. 


\section{Tabla 10}

Correlaciones por pregunta 9-12

\begin{tabular}{|c|c|c|c|c|}
\hline $\begin{array}{l}\text { Question } \\
\text { Number }\end{array}$ & & & $\begin{array}{l}\text { Score without Answer } \\
\text { Streak Bonus (points) }\end{array}$ & $\begin{array}{c}\text { Answer Time } \\
\text { (seconds) }\end{array}$ \\
\hline \multirow{6}{*}{9 Quiz } & \multirow{3}{*}{$\begin{array}{l}\text { Score without Answer } \\
\text { Streak Bonus (points) }\end{array}$} & Correlación de Pearson & 1 &,$- 192 *$ \\
\hline & & Sig.(bilateral) & &, 021 \\
\hline & & $\mathrm{N}$ & 144 & 144 \\
\hline & \multirow{3}{*}{ Answer Time (seconds) } & Correlación de Pearson &,$- 192 *$ & 1 \\
\hline & & Sig. (bilateral) &, 021 & \\
\hline & & $\mathrm{N}$ & 144 & 144 \\
\hline \multirow{6}{*}{10 Quiz } & \multirow{3}{*}{$\begin{array}{l}\text { Score without Answer } \\
\text { Streak Bonus (points) }\end{array}$} & Correlación de Pearson & 1 &,$- 259 * *$ \\
\hline & & Sig. (bilateral) & &, 002 \\
\hline & & $\mathrm{N}$ & 144 & 144 \\
\hline & \multirow{3}{*}{ Answer Time (seconds) } & Correlación de Pearson &,$- 259 * *$ & 1 \\
\hline & & Sig. (bilateral) &, 002 & \\
\hline & & $\mathrm{N}$ & 144 & 144 \\
\hline \multirow{6}{*}{11 Quiz } & \multirow{3}{*}{$\begin{array}{l}\text { Score without Answer } \\
\text { Streak Bonus (points) }\end{array}$} & Correlación de Pearson & 1 &,$- 306 * *$ \\
\hline & & Sig. (bilateral) & &, 000 \\
\hline & & $\mathrm{N}$ & 144 & 144 \\
\hline & \multirow{3}{*}{ Answer Time (seconds) } & Correlación de Pearson &,$- 306 * *$ & 1 \\
\hline & & Sig. (bilateral) &, 000 & \\
\hline & & $\mathrm{N}$ & 144 & 144 \\
\hline \multirow{6}{*}{12 Quiz } & \multirow{3}{*}{$\begin{array}{l}\text { Score without Answer } \\
\text { Streak Bonus (points) }\end{array}$} & Correlación de Pearson & 1 &,$- 351 * *$ \\
\hline & & Sig. (bilateral) & &, 000 \\
\hline & & $\mathrm{N}$ & 144 & 144 \\
\hline & \multirow{3}{*}{ Answer Time (seconds) } & Correlación de Pearson &,$- 351 * *$ & 1 \\
\hline & & Sig. (bilateral) & 000 & \\
\hline & & $\mathrm{N}$ & 144 & 144 \\
\hline
\end{tabular}

Nota. Fuente: SPSS - Elaboración propia. 


\section{Tabla 11}

Correlaciones por pregunta 13-15

\begin{tabular}{|c|c|c|c|c|}
\hline $\begin{array}{l}\text { Question } \\
\text { Number }\end{array}$ & & & $\begin{array}{l}\text { Score without Answer } \\
\text { Streak Bonus (points) }\end{array}$ & $\begin{array}{c}\text { Answer Time } \\
\text { (seconds) }\end{array}$ \\
\hline \multirow{6}{*}{13 Quiz } & \multirow{3}{*}{$\begin{array}{l}\text { Score without Answer } \\
\text { Streak Bonus (points) }\end{array}$} & Correlación de Pearson & 1 &,$- 228 * *$ \\
\hline & & Sig.(bilateral) & & ,006 \\
\hline & & $\mathrm{N}$ & 144 & 144 \\
\hline & \multirow{3}{*}{ Answer Time (seconds) } & Correlación de Pearson &,$- 228 * *$ & 1 \\
\hline & & Sig. (bilateral) &, 006 & \\
\hline & & $\mathrm{N}$ & 144 & 144 \\
\hline \multirow{6}{*}{14 Quiz } & \multirow{3}{*}{$\begin{array}{l}\text { Score without Answer } \\
\text { Streak Bonus (points) }\end{array}$} & Correlación de Pearson & 1 &,$- 437 * *$ \\
\hline & & Sig. (bilateral) & &, 000 \\
\hline & & $\mathrm{N}$ & 144 & 144 \\
\hline & \multirow{3}{*}{ Answer Time (seconds) } & Correlación de Pearson &,$- 437 * *$ & 1 \\
\hline & & Sig. (bilateral) &, 000 & \\
\hline & & $\mathrm{N}$ & 144 & 144 \\
\hline \multirow{6}{*}{15 Quiz } & \multirow{3}{*}{$\begin{array}{l}\text { Score without Answer } \\
\text { Streak Bonus (points) }\end{array}$} & Correlación de Pearson & 1 &,$- 207 *$ \\
\hline & & Sig. (bilateral) & &, 013 \\
\hline & & $\mathrm{N}$ & 144 & 144 \\
\hline & \multirow{3}{*}{ Answer Time (seconds) } & Correlación de Pearson &,$- 207 *$ & 1 \\
\hline & & Sig. (bilateral) &, 013 & \\
\hline & & $\mathrm{N}$ & 144 & 144 \\
\hline
\end{tabular}

**. La correlación es significativa en el nivel 0,01 (bilateral).

*. La correlación es significativa en el nivel 0,05 (bilateral).

Nota. Fuente: SPSS - Elaboración propia.

\section{Conclusiones}

En este trabajo, se muestra que los tiempos de respuesta proveen información sobre el pensamiento de los estudiantes al evaluar contenidos conceptuales. Para esta población estudiantil, las respuestas incorrectas toman en promedio más tiempo que las respuestas correctas, considerando que aquellas respuestas incorrectas rápidas sugieren concepciones alternativas automáticas. Dicho de otra forma, encontramos una relación velocidad-precisión invertida porque las respuestas correctas se dan más rápido que las incorrectas. 
Tomados en conjunto, estos hallazgos suponen que los tiempos de respuesta pueden ayudar a estimar la duración de una tarea en particular, más todavía en estos tiempos donde la educación virtual está en pleno crecimiento. Así pues, esta investigación también es una crítica hacia la sobreutilización del tiempo asignado para las pruebas en línea, particularmente las de tipo selección múltiple. Pues a pesar de las ventajas de esta nueva modalidad, es probable que pronto haya que realizar ajustes para proteger la integridad académica.

Asimismo, los resultados obtenidos no distan mucho de los conseguidos en estudios anteriores, pues, aunque tiempo y puntaje (rendimiento académico) no presenten correlaciones altas, su relación negativa permite comprender cómo operan los estudiantes en contextos virtuales, sobre todo a la hora de la evaluación. Esto podría justificar el hecho de que no es conveniente asignar más tiempo del que se requiere para un examen donde se evalúan contenidos conceptuales, como en este caso.

Finalmente, también está claro que la practicidad de Kahoot lo sitúa como uno de los mejores CRS del mercado, por su gratuidad, versatilidad y la disponibilidad de reportes para el análisis respectivo. Por ello, su utilización durante las evaluaciones representa una oportunidad para promover la integridad académica, ya que, al generar competencia con límite de tiempo, contribuye a disminuir los comportamientos de deshonestidad académica.

\section{Recomendaciones}

- Es importante recordar que estos hallazgos responden a la población bajo estudio, se debe tener cuidado de no generalizar demasiado, en el caso de que se desee extrapolar a otra institución.

- La relación tiempo-puntuación es un fenómeno poco estudiado en Latinoamérica, se debería poner más énfasis en esto para entender ciertos comportamientos, principalmente los vinculados a la actividad académica.

- Si bien Kahoot ha mostrado ser un aliado durante las evaluaciones virtuales, hay que procurar no caer en la sobreutilización, pues podría volverse monótono y vulnerable.

- En este trabajo se ha visto la importancia de medir los procesos académicos. Por ello, las instituciones educativas deberían otorgar relevancia a la usabilidad de los datos, pues es bien sabido que no solo Kahoot facilita la reportería, hay otros recursos que también permiten hacerlo. 


\section{Referencias Bibliográficas}

Balderas, A., \& Caballero-Hernández, J. A. (2020). Analysis of Learning Records to Detect Student Cheating on Online Exams: Case Study during COVID-19 Pandemic. TEEM'20: Eighth International Conference on Technological Ecosystems for Enhancing Multiculturality. Salamanca: Association for Computing Machinery.

BID. (20 de Mayo de 2020). Banco Interamericano de Desarrollo. Obtenido de https://publications. iadb.org/publications/spanish/document/La-educacion-superior-en-tiempos-de-COVID-19Aportes-de-la-Segunda-Reunion-del-Di\%C3\%A1logo-Virtual-con-Rectores-de-UniversidadesLideres-de-America-Latina.pdf

De Paola, M., \& Gioia, F. (2016). Who Performs Better under Time Pressure? Results from a field. Journal of Economic Psychology, 37-53.

Engelhardt, L., \& Goldhammer, F. (2019). Validating Test Score Interpretations Using Time Information. Frontiers in Psychology, 1-18.

Flores Hinostroza, E. M., Mendoza, D. J., Castillo Pinos, K. M., \& Gonzalez Sanchez, J. F. (2021). Análisis del nivel de plagio de los resultados de los exámenes online Y Su Correlación Con Los Trabajos De Titulacion De Pregrado. RECIMA21 - Revista Cientifica Multidisciplinar, 1-14.

García-Peñalvo, F., Corell, A., Abella-García, V., \& Grande, M. (2020). La evaluación online en la educación superior en tiempos de la COVID-19. Education in the Knowledge Society, 1-26.

Landrum, R. E., Carlson, H., \& Manwaring, W. (2009). The relationship between time to complete a test. Psychology Learning and Teaching, 53-56. Obtenido de https://journals.sagepub.com/ doi/pdf/10.2304/plat.2009.8.2.53

Montejo Bernardo, J. (2020). Exámenes no presenciales en época del COVID-19 y el temor al engaño. Un estudio de caso en la Universidad de Oviedo. Magister, 102-110. doi:https://doi. org/10.17811/msg.32.1.2020.102-110

Nguyen, J. G., Keuseman, K. J., \& Humston, J. J. (2020). Minimize Online Cheating for Online Assessments During COVID-19 Pandemic. Journal of Chemical Education, 97(9), 3429-3435. doi:10.1021/acs.jchemed.0c00790

Paul, C. A., \& Rosenkoetter, J. (1980). The Relationship between the Time Taken to Complete an Examination and the Test Score Received. Teaching of Psychology, 108-109.

Perea Moreno, A. J. (2018). El uso de los sistemas de respuesta interactiva como herramienta para favorecer el aprendizaje proactivo en Ingeniería. Revista de Innovación y Buenas Prácticas Docentes, 91-96. 
Rodríguez Morales, P., \& Luzardo Verde, M. (2020). Cómo asegurar evaluaciones válidas y detectar falseamiento en pruebas a distancia síncronas. Revista Digital de Investigación en Docencia Universitaria, 2.

Rojas-Castro, P. (2017). Learning Analytics: Una revisión de la literatura. Educación y Educadores, 20(1), 106-127. Obtenido de https://www.redalyc.org/pdf/834/83449754006.pdf

Terranova, C. (1972). Relationship between Test Scores and Test Time. The Journal of Experimental Education, 81-83. Obtenido de https://www.jstor.org/stable/20157287

Verleger, M. A. (2016). Just Five More Minutes: The Relationship Between Timed and Untimed Performance on an Introductory Programming Exam. 2016 ASEE Annual Conference \& Exposition. New Orleans, Louisiana. Obtenido de https://peer.asee.org/just-five-more-minutesthe-relationship-between-timed-and-untimed-performance-on-an-introductory-programmingexam

Wang, A. I., \& Tahir, R. (2020). The effect of using Kahoot! for learning - A literature review. Computers \& Education, 1-15.

Weber, J. E., Bohnen, H., \& Smith, J. A. (2011). One More Time: The Relationship Between Time Taken to Complete an Exam and the Grade Received. En BUSINESS RESEARCH YEARBOOK (Vol. XVIII, págs. 301-306). Beltsville: International Graphics. Obtenido de https://www.researchgate.net/profile/Paloma-Turnes/publication/292762859_Developing_ NFC_based_applications_and_services_Innovation_and_Research_Directions/links/5d8065a 0458515fca16e02b8/Developing-NFC-based-applications-and-services-Innovation-andResearc 